\title{
Differences in attenuation pattern in myocardial SPECT between CZT and conventional gamma
} cameras

\author{
Jenny Oddstig, PhD, , ${ }^{\mathrm{a}, \mathrm{c}}$ Elin Martinsson, MSc, ${ }^{\mathrm{b}}$ Jonas Jögi, MD, PhD, ${ }^{\mathrm{c}}$ \\ Henrik Engblom, MD, PhD, ${ }^{c}$ and Cecilia Hindorf, $\mathrm{PhD}^{\mathrm{a}}$ \\ a Department of Radiation Physics, Skåne University Hospital, Lund, Sweden \\ b Department of Medical Radiation Physics, Clinical Sciences, Lund University, Lund, Sweden \\ c Department of Clinical Physiology and Nuclear Medicine, Skåne University Hospital, Lund \\ University, Lund, Sweden
}

Received Nov 28, 2017; accepted Apr 26, 2018

doi: 10.1007/s12350-018-1296-6

Background. In myocardial perfusion imaging (MPI), single-photon emission tomography (SPECT) soft-tissue attenuation by the abdomen, breasts, and lateral chest wall may create artifacts that mimic true perfusion defects. This may cause misdiagnosis of myocardial perfusion. The aim of the present study was to compare the localization, extent, and depth of attenuation artifacts in MPI SPECT for a multi-pinhole cadmium zinc telluride (CZT) camera vs a conventional gamma camera.

Methods. Phantom and patient measurements were performed using a CZT camera (GE NM 530c) and a conventional gamma camera (GE Ventri). All images were attenuation corrected with externally acquired low-dose computed tomography. The localization, extent, and depth of the attenuation artifact were quantified by comparing attenuation-corrected and nonattenuation-corrected images.

Results. Attenuation artifacts were shifted from the inferolateral wall to the lateral wall using the CZT camera compared to a conventional camera in both the patient and the phantom. The extent of the attenuation artifact was significantly larger for the CZT camera compared to the conventional camera $(23 \pm 5 \%$ vs $15 \pm 5 \%, P<.001)$ for patients and the result was similar for the phantom (28\% vs $19 \%$ ). Furthermore, the depth of the attenuation artifact (percent of maximum counts) was less pronounced for the CZT camera than for the conventional camera, both for phantom measurements $(73 \%$ vs $67 \%)$ and patients $(72 \pm 3 \%$ vs $68 \pm 4 \%, P<.001)$.

Conclusions. Attenuation artifacts are found in different locations to different extents and depths when using a CZT camera vs a conventional gamma camera for MPI SPECT. This should be taken into consideration when evaluating MPI SPECT studies to avoid misinterpretation of myocardial perfusion distribution. (J Nucl Cardiol 2019;26:1984-91.)

Key Words: Myocardial perfusion imaging $\cdot$ SPECT $\cdot$ CZT detector $\cdot$ conventional gamma camera $\cdot$ attenuation artifact

Electronic supplementary material The online version of this article (https://doi.org/10.1007/s12350-018-1296-6) contains supplementary material, which is available to authorized users.

The authors of this article have provided a PowerPoint file, available for download at SpringerLink, which summarises the contents of the paper and is free for re-use at meetings and presentations. Search for the article DOI on SpringerLink.com.

Funding This work was partly supported by research funding from the Region of Scania, the Swedish Heart and Lung Foundation, and Lund University Faculty of Medicine.
Reprint requests: Jenny Oddstig, PhD, Department of Clinical Physiology and Nuclear Medicine, Skåne University Hospital, Lund University, 22185 Lund, Sweden; jenny.oddstig@skane.se $1071-3581 / \$ 34.00$

Copyright (c) 2018 The Author(s) 


\begin{tabular}{|c|c|}
\hline Abbreviations & \\
\hline $\mathrm{AC}$ & Attenuation corrected \\
\hline BMI & Body mass index \\
\hline CT & Computed tomography \\
\hline CZT & Multi-pinhole cadmium zinc telluride \\
\hline MLEM & $\begin{array}{l}\text { Maximum likelihood estimation } \\
\text { method }\end{array}$ \\
\hline MPI & Myocardial perfusion imaging \\
\hline NAC & Non-attenuation corrected \\
\hline OSEM & $\begin{array}{l}\text { Ordered subset expectation } \\
\text { maximization }\end{array}$ \\
\hline SD & Standard deviation \\
\hline SPECT & $\begin{array}{l}\text { Single-photon emission computed } \\
\text { tomography }\end{array}$ \\
\hline
\end{tabular}

See related editorial, pp. 1992-1995

\section{INTRODUCTION}

Myocardial perfusion imaging (MPI) with singlephoton emission computed tomography (SPECT) is the most widely used non-invasive imaging method for the detection of stress-induced ischemia and viability due to flow-limiting coronary stenosis. Traditionally, conventional MPI SPECT images have been acquired using a scintillation gamma camera with a parallel-hole collimator and a rotating gantry. Recently, a new generation of dedicated gamma cameras for MPI with cadmium zinc telluride (CZT) detectors, stationary gantry, and multi-pinhole collimators has been introduced. The agreement between multi-pinhole CZT technology and conventional technology using a NaI-crystal for visualizing myocardial perfusion has been clinically validated in several studies. ${ }^{1-4}$ CZT technology is associated with higher spatial resolution, higher sensitivity, and better energy resolution compared to conventional gamma cameras with NaI-crystals. ${ }^{5-7}$ The CZT camera system also enables complete 3D coverage of the heart using a stationary gantry. This permits sampling of the total heart volume simultaneously in only a few minutes and/ or reducing the administered activity. 8,9

The diagnostic accuracy for MPI is high, and the use of MPI SPECT for risk stratification has been wellvalidated. ${ }^{10,11}$ However, localized soft-tissue attenuation by the abdomen, breasts, and lateral chest wall may create artifacts that mimic true perfusion defects. Diagnostic specificity for coronary artery disease can be increased if attenuation correction is applied to MPI SPECT images. Attenuation correction of MPI SPECT studies for the CZT camera using X-ray-based computed tomography (CT) has been compared to attenuationcorrected MPI SPECT for conventional dual-head cameras. ${ }^{12-14}$ The results indicate that attenuation correction (AC) of MPI SPECT for CZT cameras gives the same result as that for a conventional gamma camera. However, most dedicated CZT cameras for MPI are not equipped with an integrated CT component, which is why many MPI studies using CZT cameras are performed without attenuation correction. When MPI studies are interpreted without attenuation correction, it is important to be aware of the typical attenuation pattern in order to prevent attenuation artifacts from being interpreted as perfusion abnormalities.

To what extent the attenuation patterns for the CZT camera with pinhole collimators and static gantry differ from the conventional gamma camera with parallel-hole collimators and rotating gantry is not known. Therefore, the aim of this study was to compare the localization, extent, and depth of attenuation artifacts in myocardial perfusion SPECT both in phantom experiments and in patients using the two different gamma cameras.

\section{MATERIALS AND METHODS}

\section{Phantom Measurements}

Phantom measurements were performed on both a CZT gamma camera (Discovery NM 530c; GE Healthcare, Milwaukee, WI, USA) and a conventional gamma camera (Ventri; GE Healthcare, Milwaukee, WI, USA) using an elliptical lungspine body phantom model ECT/LUNG/P with the cardiac insert model ECT/CAR/I (Data Spectrum Corporation, Durham, NC, USA). The myocardial wall of the left ventricle in the cardiac insert was filled with $0.25 \mathrm{MBq} / \mathrm{mL}(0.0066 \mathrm{mCi} /$ $\mathrm{mL}){ }^{99 \mathrm{~m}} \mathrm{Tc}$. The torso was filled with water and positioned in the gamma cameras using an external laser to obtain a repeatable position. For the CZT camera, positioning was performed to ensure that the heart was placed in the center of the camera's field of view. The phantom was imaged with both cameras with an acquisition time to obtain at least twice the number of acquired counts compared to clinical patient images. The phantom measurements were first performed with the cardiac insert homogenously filled with ${ }^{99 \mathrm{~m}} \mathrm{Tc}$. Thereafter, measurements were repeated using both cameras with a 2.1$\mathrm{cm}^{3}$ solid defect situated centrally in the anterior wall. CT for attenuation correction of the phantom images was performed using an external CT (GE Discovery PET/CT 690; GE Healthcare, Milwaukee, WI, USA).

\section{Patient Measurements}

Twenty-two patients (five females) with suspected stable ischemic heart disease were included in the study. Patient characteristics are shown in Table 1. The patients were examined using a 2-day stress-rest protocol. The stress was accomplished by an adenosine infusion, and the patients received an intravenous injection of $4 \mathrm{MBq} / \mathrm{kg}(0.11 \mathrm{mCi} / \mathrm{kg})$ body weight of ${ }^{99 \mathrm{~m}} \mathrm{Tc}$-tetrofosmin in both stress and rest. All patients were examined in a supine position with the arms over the head using both the CZT and conventional camera 
approximately 1 hour after administration of ${ }^{99 \mathrm{~m}} \mathrm{Tc}$-tetrofosmin. A CT for attenuation correction of the MPI SPECT images was obtained (Discovery PET/CT 690; GE Healthcare, Milwaukee, WI, USA) on the same day that the stress examination was performed. In total, 74 acquisitions were performed. Nineteen patients were examined using both cameras during the stress test, and 18 were examined with both cameras at rest. The study was approved by the regional ethics committee, and all patients gave their written informed consent to participate in the study.

\section{Image Acquisition and Reconstruction}

The acquisition time on the CZT camera was 480 seconds for both stress and rest. The images were reconstructed with the Maximum Likelihood Estimation Method (MLEM) algorithm using 40 iterations (Green OSL regularization alpha parameter of 0.51 and a beta of 0.3 followed by post-filtering with a Butterworth filter with a cut-off frequency of 0.37 cycles/cm and a power of 7). For the CZT camera, the voxel size was $4 \times 4 \times 4 \mathrm{~mm}$.

Table 1. Patient characteristics ( $N$ or mean \pm SD)

\section{Study population $n=22$}

\begin{tabular}{lc} 
Age years & $69 \pm 6.4(59-87)$ \\
Male & $17(77 \%)$ \\
Female & $5(23 \%)$ \\
BMI $\left(\mathrm{kg} / \mathrm{m}^{2}\right)$ & $27 \pm 3.2(21-35)$ \\
Body weight $(\mathrm{kg})$ & $84 \pm 11(62-101)$ \\
\hline
\end{tabular}

For the conventional camera, an examination was performed with the detectors in L-mode. Sixty projections were acquired at a total angular range of $180^{\circ}$ with a stop condition of 25 seconds per projection for both stress and rest. The images on the conventional gamma camera were reconstructed with a resolution recovery OSEM algorithm (Evolution, GE Healthcare) using 12 iterations and 10 subsets. This was postfiltered with a Butterworth filter with a cut-off frequency of 0.4 and a power of 10. All reconstruction parameters used followed the recommendations from the manufacturer. The voxel size for the conventional camera was $6.4 \times 6.4 \times 6.4 \mathrm{~mm}$.

All images were reconstructed both without attenuation correction and with attenuation correction using the externally acquired CT. The reconstructed images were reformatted to the standard cardiac axis format (short axis, vertical long axis, and horizontal long axis).

\section{Image Analysis}

All data were analyzed using the software Segment $\mathrm{v}$ 2.0. ${ }^{15}$ The left ventricle was segmented from the short-axis images. A fixed number of slices counted from the base (20 for the CZT and 12 for the conventional camera, depending on the voxel thickness) was analyzed. Each slice was divided into 12 equally sized sectors. The number of counts in each segment was exported to Excel and normalized to the total number of counts (Figure 1).

The ratio of the non-attenuation-corrected (NAC) image to the attenuation-corrected (AC) image from the same examination was calculated for each patient using both cameras. The same was done for the phantom measurements. The ratio was used as a descriptor of the attenuation artifacts.

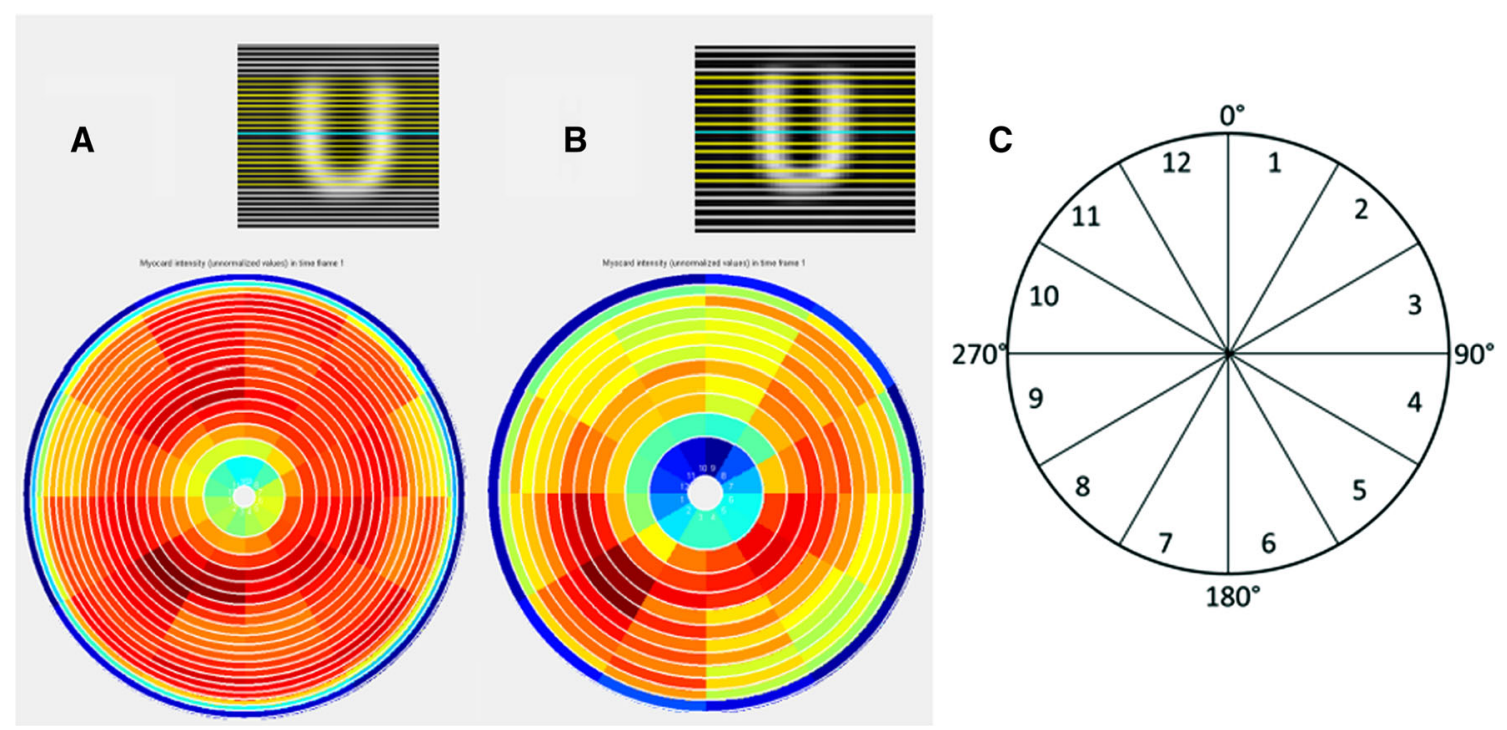

Figure 1. The segmentation of the left ventricle in $\mathbf{A}$ the multi-pinhole cadmium zinc telluride (CZT) camera with the heart volume in 20 short-axis slices, $\mathbf{B}$ the conventional camera with the heart volume in 12 short-axis slices, and $\mathbf{C}$ each short-axis slice of the left ventricle divided into 12 sectors. 


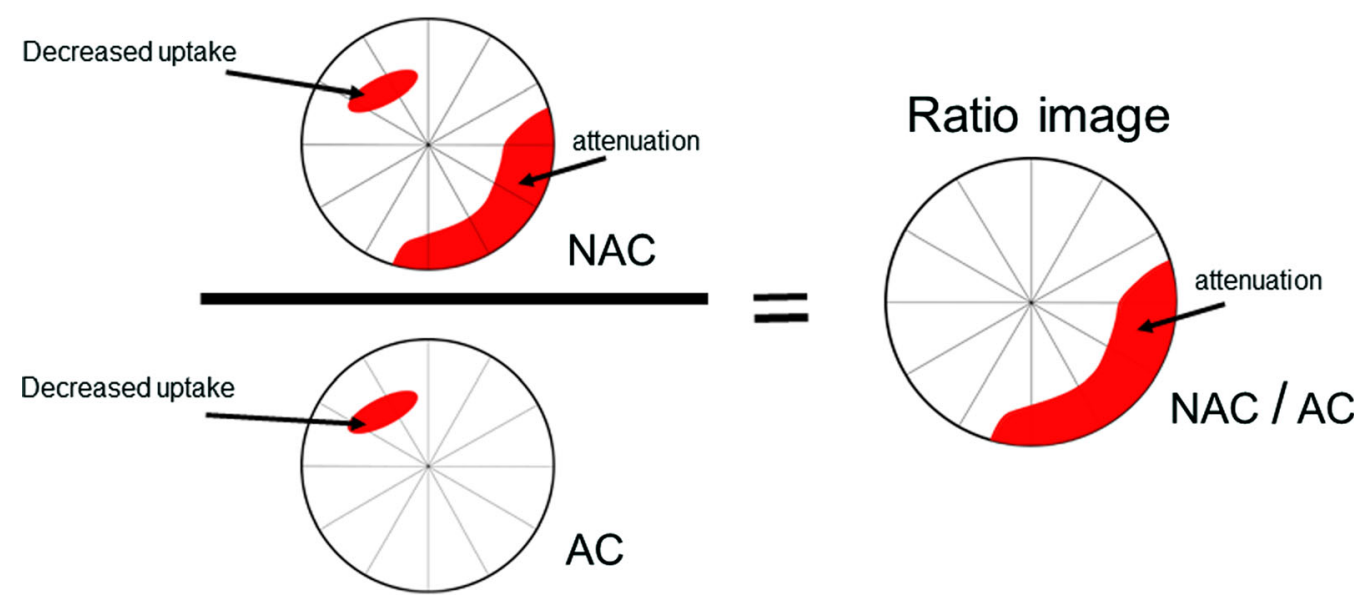

Figure 2. The ratio image of a non-attenuation-corrected (NAC) image and the attenuationcorrected (AC) image characterizing the attenuation artifacts, showing that all sectors not containing attenuation have a ratio of approximately 1 , and the sectors with attenuation have a value $<1$.

A Extent

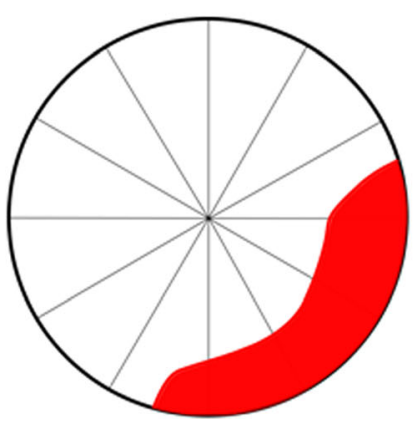

B Localization

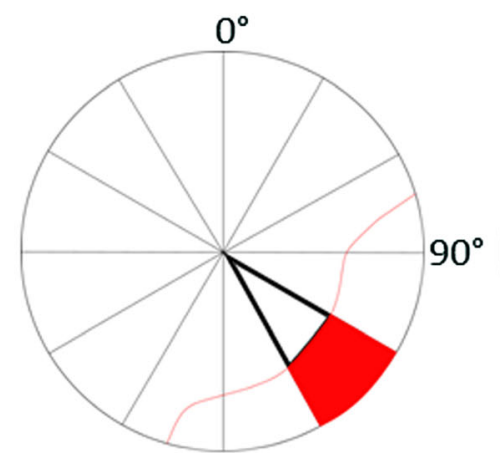

C Depth

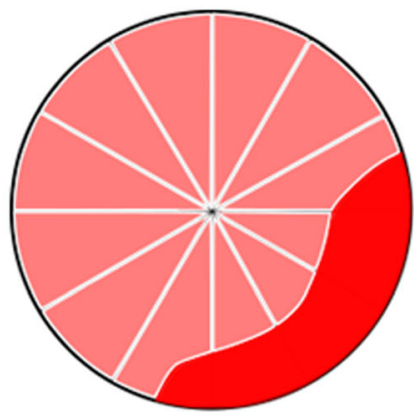

Figure 3. Three different parameters defining differences in the attenuation artifact were used to show that $\mathbf{A}$ the extent of attention artifact is the fraction of the image containing the attenuation, $\mathbf{B}$ the localization is centrum sector containing the attenuation, and $\mathbf{C}$ the depth is the reduction of counts in the area with attenuation.

For all sectors not containing any attenuation, the ratio was 1 , and sectors with attenuation had a ratio below 1 (Figure 2).

From the ratio images of the phantom, a threshold for delineation of attenuation was defined. The threshold was specified by experienced observers by finding the value at which the attenuation artifact was included, but nothing more. These thresholds, which were specific for each gamma camera, were used on NAC/AC-ratio images in patients and phantoms to determine the extent, localization, and depth of the attenuation artifact.

The extent of the attenuation artifact was determined as the number of segments in which the attenuation artifact was present divided by the total number of segments (Eq. 1). The extent was considered to be the fraction of the image containing the attenuation (Figure 3A).

$$
\text { Extent }=\frac{\text { Number of segment in the attenuation }}{\text { Total number of segments }}
$$

The localization of attenuation was defined as the angle of the attenuation artifact center according to the reference system described in Figure 3B. Furthermore, the depth of attenuation was calculated as the mean of counts in the attenuation segments divided by the mean of counts in the $30 \%$ of segments with the highest counts (Eq. 2). The depth was considered to be the reduction of counts in the area with attenuation (Figure 3C).

Depth $=\frac{\text { mean counts in the attenuation segments }}{\text { mean of counts in the } 30 \% \text { of segment with highest counts }}$. 


\section{Statistical Analysis}

The data are presented as mean \pm standard deviation (SD). A paired $t$ test was used to test for differences between the two cameras. Statistical significance was defined as $P<.05$.

\section{RESULTS}

\section{Attenuation Thresholds}

The threshold for delineation of attenuation was determined to be 0.90 and 0.85 for the CZT and conventional camera, respectively. The delineation of attenuation in the phantom experiment for both cameras is shown in Figure 4A. Attenuation correction with CT produced similar images of the phantom for the two cameras (Figure 4B). The phantom measurements with a defect in the anterior wall confirmed the attenuation shift, yielding a defect located in the same sector for both cameras, while the attenuation artifact was shifted (Figure 4C).

\section{Attenuation Localization}

The phantom measurements showed a shift in attenuation from the inferolateral wall to the lateral wall for the CZT camera compared to the conventional camera. The calculation of the localization of the attenuation artifacts showed a shift of $15^{\circ}$ and $13^{\circ}$ from the inferolateral wall to the lateral wall for the CZT camera compared to the conventional camera, respectively (Table 2). The difference between the cameras was statistically significant in patients $(P<.001)$.

\section{Attenuation Extent}

The extent of attenuation for the phantom experiment was larger in the CZT images compared to the conventional images ( $28 \%$ and $19 \%$, respectively). The corresponding value of the extent of attenuation in the acquisitions of 74 patients was $23 \pm 5 \%$ and $15 \pm 5 \%$ for the CZT images and the conventional images, respectively $(P<.001)$.

\section{Attenuation Depth}

For the phantom measurements, the depth (percent of maximum counts) within the attenuation artifact was $73 \%$ and $67 \%$ for the CZT camera and conventional gamma camera, respectively. The corresponding value of the depth of the attenuation artifact for the 74 patient acquisitions was $72 \pm 3 \%$ for the CZT camera and $68 \pm 4 \%$ for the conventional camera $(P<.001)$.

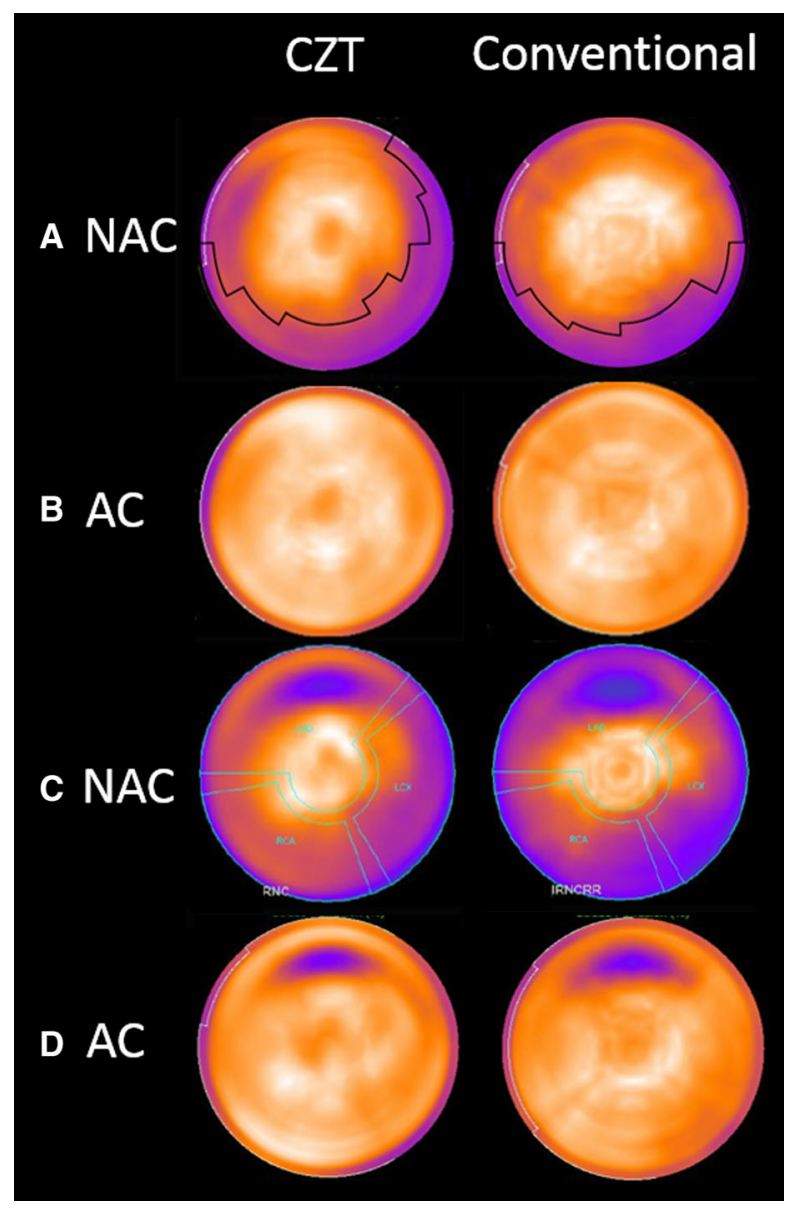

Figure 4. The polar plots of the phantom measured in the multi-pinhole cadmium zinc telluride (CZT) and conventional camera showing A non-attenuation-corrected images with the threshold value of the attenuation artifact marked with a solid line where the attenuation artifact has different patterns on the camera with different techniques, B CT attenuation-corrected (AC) images where the attenuation artifact cannot be seen, $\mathbf{C}$ a $2.1-\mathrm{cm}^{3}$ defect situated in the anterior wall where the defect appears in the same area on both cameras while the attenuation artifact has different patterns, and D CT attenuation-corrected (AC) images of the phantom with a $2.1-\mathrm{cm}^{3}$ defect in the anterior wall.

Figure 5 shows an example of one patient examined with both the CZT camera and the conventional gamma camera.

\section{DISCUSSION}

This study characterized the differences in the attenuation artifact for the CZT camera compared to a conventional gamma camera. The attenuation artifact was shifted counter-clockwise from the inferolateral wall to the lateral wall for the CZT camera. The attenuation artifact was distributed over a larger part of 
Table 2. The mean localization, extent, and depth of the attenuation artifact for phantom and patient

\section{CZT camera}

$150^{\circ}$

$155^{\circ} \pm 17^{\circ}$

$28 \%$

$23 \pm 5 \%$

$73 \%$

$72 \pm 3 \%$
Conventional camera

\section{$165^{\circ}$}

$168^{\circ} \pm 15^{\circ}$

$19 \%$

$15 \pm 5 \%$

$67 \%$

$68 \pm 4 \%$

The difference between the cameras was statistically significant in patients $(P<.001)$

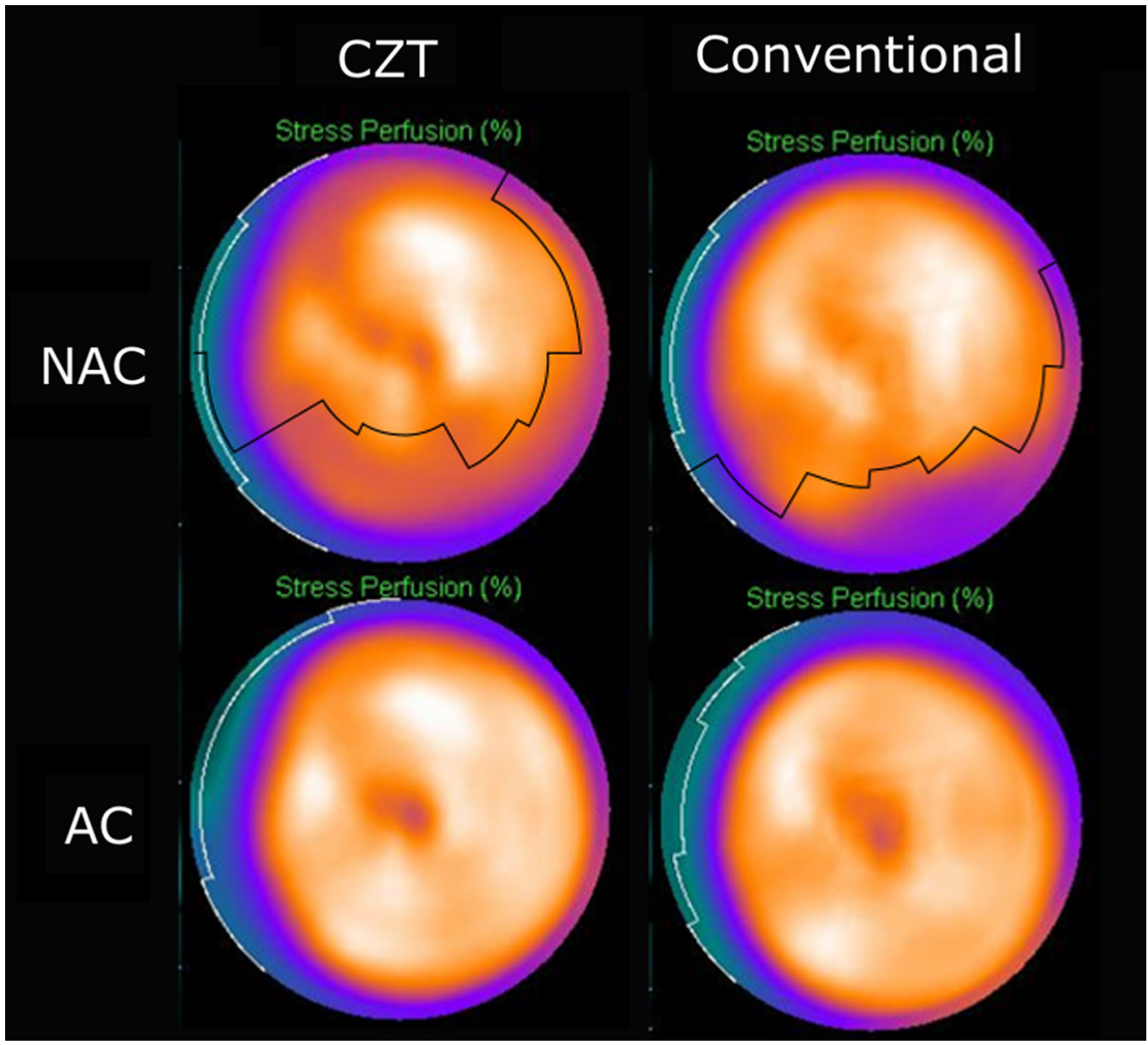

Figure 5. The NAC and AC bullseye plot images for one patient examined using both the multipinhole cadmium zinc telluride (CZT) camera and the conventional camera, showing the threshold value of the attenuation artifact marked with a solid line in the NAC images.

the image, but attenuation was less deep for the CZT camera compared to the conventional camera. The difference in attenuation localization was not caused by variations in phantom positioning because a shifted attenuation artifact also appeared in the phantom measurements with a defect, and the defect was located in the same position for both cameras. This also showed that the shift in the attenuation artifact was not an effect 
of the different linearization of the myocardium in the reconstruction. Both the phantom and the patients were accurately positioned within the camera's field of view to avoid positioning defects that have been shown to occur with the CZT camera under suboptimal positioning. ${ }^{16}$

The difference in attenuation artifact patterns between the CZT and conventional gamma camera may arise from the difference in the measurement geometry. Using a parallel-hole collimator in the conventional camera, the detected photons are emitted perpendicular to the detector for all pixels in all 60 projections at $180^{\circ}$ around the patient. The CZT camera measures the object with 19 stationary pinhole collimators arranged in three rows. The detectors in the central row are directed at the same angle as that for the conventional camera with the parallel-hole collimator. The detectors located on each side of the central row are rotated against the center of the detected volume. The use of pinhole collimators and the rotated detectors produces a different image for the CZT camera compared to the parallel-hole collimator. It also generates other distance angles with respect to the myocardium, which is why the attenuation artifacts may occur to different extents. The parallax error for the pinhole collimators may also be a contributing factor for the difference in the attenuation artifact patterns between the cameras.

The use of CTs for attenuation correction (AC) maps from another scanner has previously been useful for attenuation correction of MPI SPECT as long as the co-registration of all image stacks was previewed. ${ }^{17-19}$ The same result was obtained for the CZT camera and for the conventional cameras. The NAC/AC ratio can therefore be used to analyze the difference in attenuation artifact patterns obtained using the different cameras.

The calculation for the relative depth for the phantom measurement concluded that the attenuation artifact was deeper in the conventional camera compared to the CZT camera (67\% and 73\%, respectively). The patient measurements correlated well with the phantom results $(68 \pm 4 \%$ and $72 \pm 3 \%[P<.05]$ for the conventional and CZT cameras, respectively). The results in the current study show less isotope reduction in the attenuation artifact for the CZT camera compared to the conventional gamma camera, which supports the findings of Liu et al. ${ }^{20}$

The extent of the attenuation artifact differed between the CZT camera and the conventional camera for both the phantom measurements and those in patients. Thus, it is important for physicians interpreting MPI images to be aware of the differences in attenuation patterns for conventional and CZT pinhole gamma cameras when evaluating non-attenuation-corrected images so that they do not overestimate uptake reductions in the lateral wall or underestimate uptake reductions in the inferolateral wall, producing falsepositive or false-negative results.

\section{LIMITATIONS}

Due to the predominant number of males in the patient population with mostly inferior wall attenuation, it is not possible to draw any conclusions regarding the anterior attenuation artifacts arising in women as a result of breast attenuation. The clinical impact of these findings has not been studied here because not all patients were examined using both cameras under both stress and rest conditions. Thus, this report should be considered a proof-of-concept study of different attenuation patterns for CZT cameras compared to conventional cameras. Differences between patients and phantoms might be affected by basing the threshold on experienced observer opinions. This was, however, the only way to approach this study given the lack of any true reference standard. The use of mean counts over a large area might lessen the impact of more focal attenuation artifacts; however, this results in a method not influenced by an observer.

\section{NEW KNOWLEDGE GAINED}

This study establishes differences in the appearance of inferior attenuation artifacts for the CZT camera (GE Discovery NM 530c) compared to the conventional gamma camera (GE Ventri). It is important to consider these differences when interpreting data and especially when comparing images from different cameras so that one does not over- or underestimate perfusion defects in the myocardium.

\section{CONCLUSIONS}

Attenuation artifacts are produced in different locations to varying extents and depths when comparing a multi-pinhole cadmium zinc telluride (CZT) camera to a conventional gamma camera for MPI SPECT. This needs to be taken into consideration when evaluating MPI studies to avoid misinterpreting the location and extent of a myocardial perfusion distribution.

\section{Acknowledgements}

The authors would like to thank Christel Kullberg and Berit Olsson for excellent technical assistance. Financial support was provided by the Swedish Heart Lung Foundation, Lund University Faculty of Medicine, and the Region of Scania. 


\section{Disclosure}

None of the authors have any disclosures to declare.

\section{Open Access}

This article is distributed under the terms of the Creative Commons Attribution 4.0 International License (http:// creativecommons.org/licenses/by/4.0/), which permits unrestricted use, distribution, and reproduction in any medium, provided you give appropriate credit to the original author $(s)$ and the source, provide a link to the Creative Commons license, and indicate if changes were made.

\section{References}

1. Buechel RR, Herzog BA, Husmann L, Burger IA, Pazhenkottil AP, Treyer $\mathrm{V}$, et al. Ultrafast nuclear myocardial perfusion imaging on a new gamma camera with semiconductor detector technique: First clinical validation. Eur J Nucl Med Mol Imaging 2010;37:773-8.

2. Esteves FPRP, Folks RD, Keidar Z, Askew JW, Rispler S, O'Connor MK, et al. Novel solid-state-detector dedicated cardiac camera for fast myocardial perfusion imaging: Multicenter comparison with standard dual detector cameras. J Nucl Cardiol 2009; 16:927-34.

3. Herzog BA, Buechel RR, Katz R, Brueckner M, Husmann L, Burger IA, et al. Nuclear myocardial perfusion imaging with a cadmium-zinc-telluride detector technique: Optimized protocol for scan time reduction. J Nucl Med 2010;51:46-51

4. Sharir T, Ben-Haim S, Merzon K, Prochorov V, Dickman D, Berman DS. High-speed myocardial perfusion imaging initial clinical comparison with conventional dual detector anger camera imaging. JACC Cardiovasc Imaging 2008;1:156-63.

5. Bocher M, Blevis IM, Tsukerman L, Shrem Y, Kovalski G, Volokh L. A fast cardiac gamma camera with dynamic SPECT capabilities: Design, system validation and future potential. Eur J Nucl Med Mol Imaging 2010;37:1887-902.

6. Gambhir SS, Berman DS, Ziffer J, Nagler M, Sandler M, Patton J, et al. A novel high-sensitivity rapid-acquisition single-photon cardiac imaging camera. J Nucl Med 2009;50:635-43.

7. Garcia EV, Faber TL, Esteves FP. Cardiac dedicated ultrafast SPECT cameras: New designs and clinical implications. J Nucl Med 2011;52:210-7.

8. Duvall WL, Croft LB, Godiwala T, Ginsberg E, George T, Henzlova MJ. Reduced isotope dose with rapid SPECT MPI imaging: Initial experience with a CZT SPECT camera. J Nucl Cardiol 2010;17:1009-14.
9. Oddstig J, Hedeer F, Jögi J, Carlsson M, Hindorf C, Engblom H. Reduced administered activity, reduced acquisition time, and preserved image quality for the new CZT camera. J Nucl Cardiol 2013;20:38-44.

10. Hachamovitch R, Berman DS, Kiat H, Cohen I, Cabico JA, Friedman J, Diamond GA. Exercise myocardial perfusion SPECT in patients without known coronary artery disease: Incremental prognostic value and use in risk stratification. Circulation 1996;93:905-14.

11. Iskander S, Iskandrian AE. Risk assessment using single-photon emission computed tomographic technetium-99m sestamibi imaging. J Am Coll Cardiol 1998;32:57-62.

12. Herzog BA, Buechel RR, Husmann L, Pazhenkottil AP, Burger IA, Wolfrum M, et al. Validation of CT attenuation correction for high-speed myocardial perfusion imaging using a novel cadmiumzinc-telluride detector technique. J Nucl Med 2010;51:1539-44.

13. van Dijk JD, Mouden M, Ottervanger JP, van Dalen JA, Knollema $\mathrm{S}$, Slump $\mathrm{CH}$, et al. Value of attenuation correction in stress-only myocardial perfusion imaging using CZT-SPECT. J Nucl Cardiol 2017;24:395-401.

14. Caobelli F, Akin M, Thackeray JT, Brunkhorst T, Widder J, Berding G, et al. Diagnostic accuracy of cadmium-zinc-telluridebased myocardial perfusion SPECT: Impact of attenuation correction using a co-registered external computed tomography. Eur Heart J Cardiovasc Imaging 2016;17:1036-43.

15. Heiberg E, Sjögren J, Ugander M, Carlsson M, Engblom H, Arheden $\mathrm{H}$. Design and validation of segment-freely available software for cardiovascular image analysis. BMC Med Imaging 2010;10:1.

16. Hindorf C, Oddstig J, Hedeer F, Hansson MJ, Jögi J, Engblom H. Importance of correct patient positioning in myocardial perfusion SPECT when using a CZT camera. J Nucl Cardiol 2014;21:695-702.

17. Goetze S, Brown TL, Lavely WC, Zhang Z, Bengel FM. Attenuation correction in myocardial perfusion SPECT/CT: Effects of misregistration and value of reregistration. $J$ Nucl Med 2007;48:1090-5.

18. Goetze S, Wahl RL. Prevalence of misregistration between SPECT and CT for attenuation-corrected myocardial perfusion SPECT. J Nucl Cardiol 2007;14:200-6.

19. Schepis T, Gaemperli O, Koepfli P, Ruegg C, Burger C, Leschka $\mathrm{S}$, et al. Use of coronary calcium score scans from stand-alone multislice computed tomography for attenuation correction of myocardial perfusion SPECT. Eur J Nucl Med Mol Imaging 2007;34:11-9.

20. Liu CJ, Cheng JS, Chen YC, Huang YH, Yen RF. A performance comparison of novel cadmium-zinc-telluride camera and conventional SPECT/CT using anthropomorphic torso phantom and water bags to simulate soft tissue and breast attenuation. Ann Nucl Med 2015;29:342-50. 